\title{
Reverse Time Migration of Prism Waves for Salt Flank Delineation
}

Wei Dai ${ }^{1}$ and Gerard T. Schuster ${ }^{2}$

${ }^{1}$ Formerly at King Abdullah University of Science and Technology; Presently at WesternGeco.

${ }^{2}$ King Abdullah University of Science and Technology.

\section{SUMMARY}

In this paper, we present a new reverse time migration method for imaging salt flanks with prism wave reflections. It consists of four steps: (1) migrating the seismic data with conventional RTM to give the RTM image; (2) using the RTM image as a reflectivity model to simulate source-side reflections with the Born approximation; (3) zero-lag correlation of the source-side reflection wavefields and receiver-side wavefields to produce the prism wave migration image; and (4) repeating steps 2 and 3 for the receiver-side reflections. An advantage of this method is that there is no need to pick the horizontal reflectors prior to migration of the prism waves. It also separately images the vertical structures at a different step to reduce crosstalk interference. The disadvantage of prism wave migration algorithm is that its computational cost is twice that of conventional RTM. The empirical results with a salt model suggest that prism wave migration can be an effective method for salt flank delineation in the absence of diving waves.

\section{INTRODUCTION}

Vertical structures such as salt flanks are usually not illuminated by primary reflections and so cannot be well imaged by conventional migration methods (Hale et al., 1992). If on the other hand strong diving waves are present, they can be reflected from the salt flank, recorded on the surface, and migrated by a two-way migration method, such as Kirchhoff migration (Ratcliff et al., 1991, 1992) or reverse time migration (RTM) (Baysal et al., 1983; McMechan, 1983; Whitmore, 1983). Even a one-way migration method can be modified (Hale et al., 1992) to incorporate diving waves for salt flank imaging.

If the diving wave is not extant due to the absence of a strong velocity gradient or a limited recording aperture, prism waves can be migrated to illuminate vertical reflectors. A prism wave is defined to be a doubly scattered wave from, typically, a vertical reflector, as illustrated by the ray diagram in Figure $1 \mathrm{a}$.

With reverse time migration, the migration of the prism waves can be accommodated in the process by embedding the subhorizontal reflection boundaries in the velocity model (Jones et al., 2007). However, incorporating the sharp boundaries into the velocity model is not trivial, and the complex migration velocity will excite complex wavefields that lead to artifacts in the RTM images (Liu et al., 2011). Another problem is that prism waves are doubly scattered waves, which are usually weaker than primaries, so that the contribution from the prism waves might be weak. In this paper, we propose a new RTM method for migrating the prism waves separately from the other reflectors by utilizing the migration image from conventional RTM.

\section{THEORY}

In the frequency domain, reverse time migration of a shot gather $d\left(\mathbf{x}_{g} \mid \mathbf{x}_{s}\right)$ can be expressed as

$$
m_{m i g}\left(\mathbf{x} \mid \mathbf{x}_{s}\right)=\sum_{\omega} \sum_{g} \omega^{2} W^{*}(\omega) G^{*}\left(\mathbf{x} \mid \mathbf{x}_{S}\right) G^{*}\left(\mathbf{x} \mid \mathbf{x}_{g}\right) d\left(\mathbf{x}_{g} \mid \mathbf{x}_{s}\right),
$$

where $m_{m i g}\left(\mathbf{x} \mid \mathbf{x}_{s}\right)$ is the migration image of the shot at $\mathbf{x}_{s}$, $W(\omega)$ is the source spectrum, $\mathbf{x}_{g}$ indicates the receiver location, $G\left(\mathbf{x} \mid \mathbf{x}_{S}\right)$ is the Green's function from a source at $\mathbf{x}_{S}$ to $\mathbf{x}$; This Green's function is computed by a finite-difference solution to the wave equation. The $*$ indicates complex conjugate. For simplicity, the angular frequency $\omega$ is silent in the notation of the Green's function $G$ and data function $d$.

For the velocity model in Figure 1a, the recorded data contain prism waves. The yellow arrows in Figure 1a indicate the ray path for a prism wave excited at $(x, z)=(4.5,0) \mathrm{km}$ and recorded at $(x, z)=(2.5,0) \mathrm{km}$, and Figure $1 \mathrm{~b}$ depicts the wavepath (Luo and Schuster, 1991) of the prism wave generated by a source with a $20-\mathrm{Hz}$ Ricker wavelet. The recorded

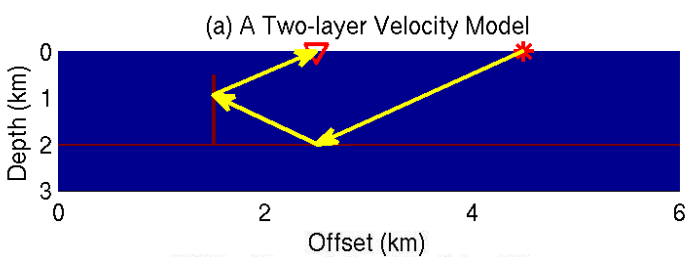

(b) The Wave Path of the Prism Wave

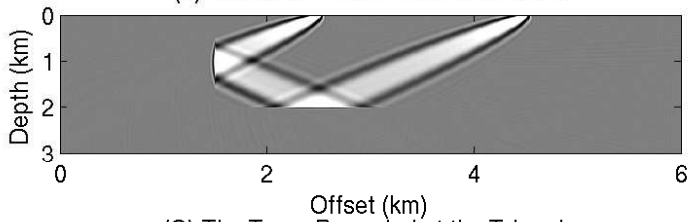

(C) The Trace Recorded at the Triangle

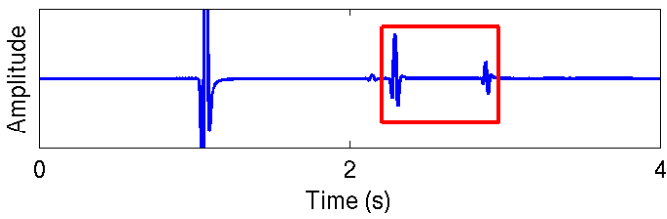

Figure 1: (a) A velocity model with a horizontal reflector and a vertical reflector. The yellow arrows indicate the ray path for a prism wave from the source at the star to the receiver at the triangle; (b) The wave path of the prism wave with a 20$\mathrm{Hz}$ Ricker wavelet; and (c) The trace recorded at the triangle. The two arrivals in the red window are the reflections from the horizontal reflector and the prism wave in panel (b).

trace is plotted in Figure 1(c) with a red window outlining the reflection from the horizontal reflector and the prism wave. For 


\section{Prism Waves RTM}

simplicity, we mute the direct wave and diffractions from the trace to keep only the part in the red window

$$
d\left(\mathbf{x}_{g} \mid \mathbf{x}_{S}\right)=d_{1}\left(\mathbf{x}_{g} \mid \mathbf{x}_{S}\right)+d_{2}\left(\mathbf{x}_{g} \mid \mathbf{x}_{S}\right),
$$

where $d_{1}\left(\mathbf{x}_{g} \mid \mathbf{x}_{S}\right)$ and $d_{2}\left(\mathbf{x}_{g} \mid \mathbf{x}_{S}\right)$ denote the first-order scattering reflection wave and the doubly scattered prism wave, respectively. When the horizontal reflector is extracted from the migration images and embedded in the migration velocity model (Figure 2a), conventional RTM can correctly migrate the prism waves to image the vertical reflector (Jones et al., 2007). In this case, the Green's function calculated with the migration velocity in Figure 2a contains two arrivals: a direct wave arrival and a reflection from the horizontal reflector Therefore, the Green's functions in equation 1 can be decomposed into two parts: $G\left(\mathbf{x} \mid \mathbf{x}_{s}\right)=G_{o}\left(\mathbf{x} \mid \mathbf{x}_{s}\right)+G_{1}\left(\mathbf{x} \mid \mathbf{x}_{s}\right)$, and $G\left(\mathbf{x} \mid \mathbf{x}_{g}\right)=G_{o}\left(\mathbf{x} \mid \mathbf{x}_{g}\right)+G_{1}\left(\mathbf{x} \mid \mathbf{x}_{g}\right)$, where $G_{o}$ and $G_{1}$ denote the direct and the reflected waves, respectively. Note that in this case $G_{o}$ is a downgoing wave and $G_{1}$ is an upgoing wave.

When the data in the red window of Figure 1(c) are migrated with the velocity model in Figure 2a, the migration image is shown in Figure 2b, and is mathematically described by

$$
\begin{aligned}
& m_{m i g}\left(\mathbf{x} \mid \mathbf{x}_{s}\right)=\sum_{\omega} \omega^{2} W^{*}(\omega)\left[G_{o}^{*}\left(\mathbf{x} \mid \mathbf{x}_{s}\right)+G_{1}^{*}\left(\mathbf{x} \mid \mathbf{x}_{s}\right)\right] \\
& {\left[G_{o}^{*}\left(\mathbf{x} \mid \mathbf{x}_{g}\right)+G_{1}^{*}\left(\mathbf{x} \mid \mathbf{x}_{g}\right)\right]\left[d_{1}\left(\mathbf{x}_{g} \mid \mathbf{x}_{s}\right)+d_{2}\left(\mathbf{x}_{g} \mid \mathbf{x}_{s}\right)\right]} \\
& =\overbrace{\sum_{\omega} \omega^{2} W^{*}(\omega) G_{o}^{*}\left(\mathbf{x} \mid \mathbf{x}_{S}\right) G_{o}^{*}\left(\mathbf{x} \mid \mathbf{x}_{g}\right) d_{1}\left(\mathbf{x}_{g} \mid \mathbf{x}_{S}\right)}^{\text {First Ellipse } O(r)} \\
& +\overbrace{\sum_{\omega} \omega^{2} W^{*}(\omega) G_{o}^{*}\left(\mathbf{x} \mid \mathbf{x}_{S}\right) G_{o}^{*}\left(\mathbf{x} \mid \mathbf{x}_{g}\right) d_{2}\left(\mathbf{x}_{g} \mid \mathbf{x}_{S}\right)}^{\text {Second Ellipse } O\left(r^{2}\right)} \\
& +\overbrace{\sum_{\omega} \omega^{2} W^{*}(\omega) G_{1}^{*}\left(\mathbf{x} \mid \mathbf{x}_{S}\right) G_{o}^{*}\left(\mathbf{x} \mid \mathbf{x}_{g}\right) d_{1}\left(\mathbf{x}_{g} \mid \mathbf{x}_{S}\right)}^{\text {Left Rabbit Ear } O\left(r^{2}\right)} \\
& +\overbrace{\sum_{\omega} \omega^{2} W^{*}(\omega) G_{o}^{*}\left(\mathbf{x} \mid \mathbf{x}_{S}\right) G_{1}^{*}\left(\mathbf{x} \mid \mathbf{x}_{g}\right) d_{1}\left(\mathbf{x}_{g} \mid \mathbf{x}_{S}\right)}^{\text {Right Rabbit Ear } O\left(r^{2}\right)} \\
& +\overbrace{\sum_{\omega} \omega^{2} W^{*}(\omega) G_{1}^{*}\left(\mathbf{x} \mid \mathbf{x}_{S}\right) G_{o}^{*}\left(\mathbf{x} \mid \mathbf{x}_{g}\right) d_{2}\left(\mathbf{x}_{g} \mid \mathbf{x}_{S}\right)}^{\text {First Prism Wave Kernel } O\left(r^{3}\right)} \\
& +\overbrace{\sum_{\omega} \omega^{2} W^{*}(\omega) G_{o}^{*}\left(\mathbf{x} \mid \mathbf{x}_{S}\right) G_{1}^{*}\left(\mathbf{x} \mid \mathbf{x}_{g}\right) d_{2}\left(\mathbf{x}_{g} \mid \mathbf{x}_{S}\right)}^{\text {Second Prism Wave Kernel } O\left(r^{3}\right)} \\
& +\quad \text { other terms. }
\end{aligned}
$$

Note that the summation over the receiver $g$ is omitted because there is only one trace in this example. With the assumption that the reflection coefficient is the angle-independent value $r$, the amplitude of the direct wave Green's function $G_{o}$ is on the order of $O(1)$ and the amplitude of the reflection wave $G_{1}$ is on the order of $O(r)$. Similarly, $d_{1}$ is with strength of $O(r)$. The prism wave $d_{2}$ is a doubly scattered wave and its amplitude is on the order $O\left(r^{2}\right)$. As an example, the first prism wave term in equation 7 has $O\left(r^{3}\right)$ because it is a product of the $d_{2}$ term with amplitude $O\left(r^{2}\right)$ and the migration kernel $G_{1} \times G_{o}$ with strength $O(r)$. With these assumptions, the amplitude of each term in the above equation can be expressed in terms of $r$ as shown in the labels.

(a) The Velocity Model with a Horizontal Reflector

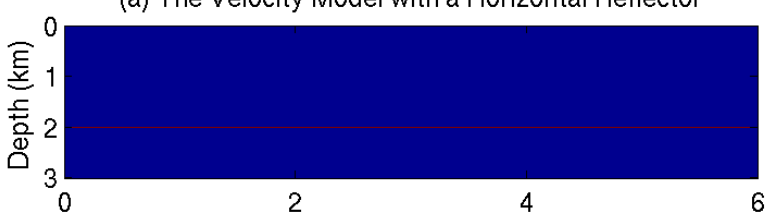

(b) Migration Image Obtained with Inhomogeneous Velocity

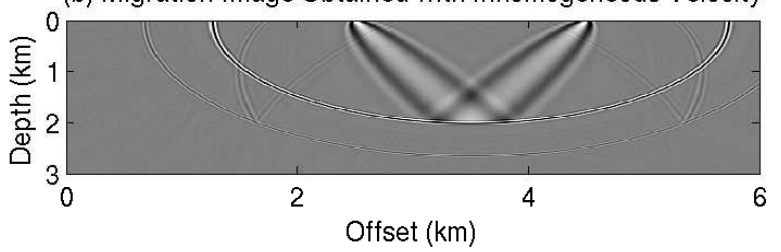

Figure 2: (a) The homogeneous velocity $(2 \mathrm{~km} / \mathrm{s})$ with a horizontal reflector embedded $(2.5 \mathrm{~km} / \mathrm{s})$; (b) The migration image of the data within the red window in Figure 1c with the velocity model in panel (a).

Figure $2 b$ shows two ellipses. The first one corresponds to the migration kernel in equation 3 with the strongest amplitude $O(r)$. When the prism wave is migrated as a primary wave (the term in equation 4), it shows up as the second ellipse in Figure $2 \mathrm{~b}$ with an amplitude $O\left(r^{2}\right)$. This ellipse is an artifact. The migration kernels in equations 5 and 6 correspond to these two "rabbit ears" with the strength $O\left(r^{2}\right)$. Equations 7 and 8 contain the migration kernels for the prism waves corresponding to these near-vertical curves in Figure $2 \mathrm{~b}$ and their amplitudes are on the order of $O\left(r^{3}\right)$, which are much weaker than other kernels, so in the migration image, the vertical reflector is of weaker amplitude compared to the horizontal ones.

If the migration kernels in equations 7 and 8 can be computed directly, the prism waves can be directly migrated without crosstalk interference. In the following section, frequency domain formulas are used for mathematical simplicity, but the numerical calculation is actually computed in the time domain by a finite-difference solution to the space-time acoustic wave equation. Given a smooth migration velocity (homogeneous velocity in this example) and a migration image of the horizontal reflector, the Green's function for the reflected wave can be computed with the Born approximation (Beylkin, 1985; Stolt and Benson, 1986) by a Born modeling operator

$$
G_{1}\left(\mathbf{x} \mid \mathbf{x}_{S}\right)=\int_{\mathbf{x}^{\prime}} \omega^{2} m_{1}\left(\mathbf{x}^{\prime}\right) G_{o}\left(\mathbf{x}^{\prime} \mid \mathbf{x}_{S}\right) G_{o}\left(\mathbf{x}^{\prime} \mid \mathbf{x}\right) d \mathbf{x}^{\prime}
$$

where $m_{1}\left(\mathbf{x}^{\prime}\right)$ is the reflectivity model representing the horizontal reflector, and the Green's function $G_{O}$ is calculated using the migration velocity. Plugging equation 9 into equation 


\section{Prism Waves RTM}

7, we get

$$
\begin{aligned}
m_{m i g}\left(\mathbf{x} \mid \mathbf{x}_{S}\right)= & \sum_{\omega} \omega^{2} W^{*}(\omega) \int_{\mathbf{x}^{\prime}} \omega^{2} m_{1}\left(\mathbf{x}^{\prime}\right) G_{o}^{*}\left(\mathbf{x}^{\prime} \mid \mathbf{x}_{s}\right) \\
& G_{o}^{*}\left(\mathbf{x}^{\prime} \mid \mathbf{x}\right) d \mathbf{x}^{\prime} G_{o}^{*}\left(\mathbf{x} \mid \mathbf{x}_{g}\right) d_{2}\left(\mathbf{x}_{g} \mid \mathbf{x}_{S}\right) \\
= & \sum_{\omega} \omega^{2} \int_{\mathbf{x}^{\prime}} \omega^{2} W^{*}(\omega) G_{o}^{*}\left(\mathbf{x}^{\prime} \mid \mathbf{x}_{S}\right) m_{1}\left(\mathbf{x}^{\prime}\right) \\
& G_{o}^{*}\left(\mathbf{x}^{\prime} \mid \mathbf{x}\right) d \mathbf{x}^{\prime} G_{o}^{*}\left(\mathbf{x} \mid \mathbf{x}_{g}\right) d_{2}\left(\mathbf{x}_{g} \mid \mathbf{x}_{S}\right) \\
= & \sum_{\omega} \omega^{2}\left[P_{1}\left(\mathbf{x} \mid \mathbf{x}_{S}\right)\right]^{*}\left[Q_{o}\left(\mathbf{x} \mid \mathbf{x}_{S}\right)\right]
\end{aligned}
$$

with

$$
\begin{aligned}
P_{1}\left(\mathbf{x} \mid \mathbf{x}_{S}\right) & =\int_{\mathbf{x}^{\prime}} \omega^{2} W(\omega) G_{o}\left(\mathbf{x}^{\prime} \mid \mathbf{x}_{S}\right) m_{1}\left(\mathbf{x}^{\prime}\right) G_{o}\left(\mathbf{x}^{\prime} \mid \mathbf{x}\right) d \mathbf{x}^{\prime} \\
Q_{o}\left(\mathbf{x} \mid \mathbf{x}_{S}\right) & =G_{o}^{*}\left(\mathbf{x} \mid \mathbf{x}_{g}\right) d_{2}\left(\mathbf{x}_{g} \mid \mathbf{x}_{S}\right) .
\end{aligned}
$$

Numerically, $P_{1}\left(\mathbf{x} \mid \mathbf{x}_{S}\right)$ are computed with two finite-difference simulations in the time domain to solve the following two equations

$$
\begin{gathered}
\left(\nabla^{2}+\omega^{2} s_{o}^{2}(\mathbf{x})\right) P_{o}\left(\mathbf{x} \mid \mathbf{x}_{s}\right)=-W(\omega) \delta\left(\mathbf{x}-\mathbf{x}_{S}\right) \\
\left(\nabla^{2}+\omega^{2} s_{o}^{2}(\mathbf{x})\right) P_{1}\left(\mathbf{x} \mid \mathbf{x}_{s}\right)=-\omega^{2} m_{1}(\mathbf{x}) P_{o}\left(\mathbf{x} \mid \mathbf{x}_{S}\right),
\end{gathered}
$$

where the slowness $s_{O}(\mathbf{x})$ is the reciprocal of the migration velocity model. The receiver-side wavefield $Q_{o}\left(\mathbf{x} \mid \mathbf{x}_{S}\right)$ can be computed by solving

$$
\left(\nabla^{2}+\omega^{2} s_{o}^{2}(\mathbf{x})\right) Q_{o}\left(\mathbf{x} \mid \mathbf{x}_{S}\right)=-d_{2}\left(\mathbf{x}_{g} \mid \mathbf{x}_{S}\right) \delta\left(\mathbf{x}-\mathbf{x}_{g}\right) .
$$

Note the wavefield propagates backward in time when solving the above equation in the time domain with the finitedifference method. When there is more than one trace in the shot gather, all the traces act as source wavelets of point sources at their respective recording locations, which implies a summation over the receiver $g$. In summary, prism wave migration requires three finite-difference simulations (equations 12, 13, and 14) to calculate the image corresponding to the term in equation 7 .

The term in equation 8 can be computed in a similar way. In summary, it requires four finite-difference simulations in total. Compared to conventional RTM, its computational cost is doubled. The advantages of this approach are as follows: (1) It avoids modifying the migration velocity as in conventional RTM of prism waves; (2) vertical structures are imaged in a separate step and reduces the crosstalk interference between different phases.

\section{NUMERICAL RESULTS}

Prism wave RTM can be used to delineate the vertical boundaries of a salt flank. In the velocity model shown in Figure 3a, an irregular salt body is placed along the left boundary. The model size is $601 \times 601$ points with a $10 \mathrm{~m}$ grid interval. The seismic survey contains 301 shots fired at a depth of $10 \mathrm{~m}$ with an even $x$-sampling of $20 \mathrm{~m}$. Every shot is recorded with 601 receivers at a $10 \mathrm{~m}$ depth and a $10 \mathrm{~m}$ receiver interval along the $x$-axis. In this case, the velocity gradient is not strong enough to generate diving waves for the short recording aperture of a $6 \mathrm{~km}$ long receiver array.

The 301 shot gathers are migrated with the smooth migration velocity in Figure $3 \mathrm{~b}$ by a conventional RTM method, and the result is shown in Figure 4a. This image clearly illuminates the subhorizontal reflectors, but only a few diffractors are visible along the salt flank.

The prism wave migration method uses the smooth migration velocity (Figure 3b) and the conventional RTM image (Figure 4a) to image the salt flank so that modification of the migration velocity is avoided. Figure $4 \mathrm{~b}$ shows the prism wave migration image, where the salt flank is clearly imaged with strong amplitudes. However, this image contains some strong artifacts originated from Figure 4a, marked by white arrows. When these artifacts in Figure $4 \mathrm{a}$ were treated as real reflectors, additional artifacts were generated in Figure 4b, which are also marked by white arrows.

To further improve the image quality, we apply a dip filter to Figure 4a to keep only the subhorizontal reflectors, and the result is shown in Figure 5a. Then, the proposed method is applied with the filtered image and the smooth velocity model to migrate the prism waves to produce the image in Figure $5 b$, which contains fewer artifacts compared to Figure 4b. Figure 6a shows the image in Figure 5b after dip filtering to keep only the subvertical reflectors. The final image is produced by summation of the migration images in Figures $5 \mathrm{a}$ and $6 \mathrm{a}$ to give Figure $6 \mathrm{~b}$, which is the migration image with the best quality. Theoretically, these two partial images should be weighted according true reflection coefficients of the horizontal and vertical reflectors. However, estimation of true reflection coefficients is not under the scope of this paper. Currently, the weighting factors for both partial images are chosen empirically.

\section{DISCUSSION AND CONCLUSION}

In this paper, we proposed a new method for migrating prism waves by RTM. There are two steps to the method: (1) Conventional RTM is applied to the data to estimate the geometry of the horizontal reflectors near the salt flank; (2) Prism wave RTM is applied to the data again, except the prism imaging condition is used rather than the conventional one. Dip filtering can also be applied to the images to reduce noise. In the example of the salt model, the salt flank can be imaged by embedding the horizontal reflectors in the velocity model, which is not trivial, but the best image is obtained by summation of two dip filtered partial migration images: one from conventional RTM and the other from the migration of the prism waves. The disadvantage of prism wave RTM is that its computational cost is twice that of conventional RTM. The empirical results suggest that the proposed method can migrate the prism waves correctly to delineate salt flanks and improve the image quality with the help of dip filtering. 


\section{Prism Waves RTM}

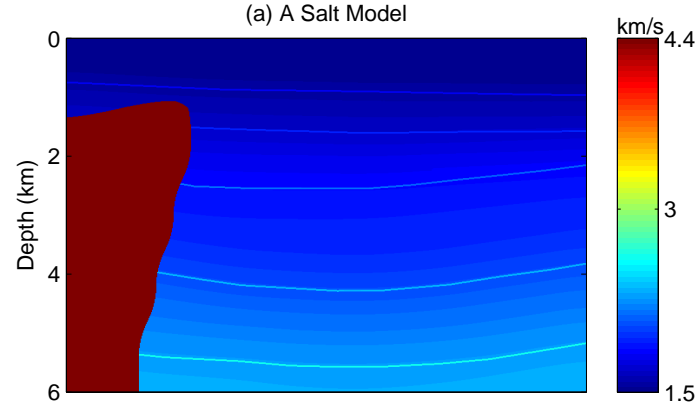

(b) Smooth Migration Velocity

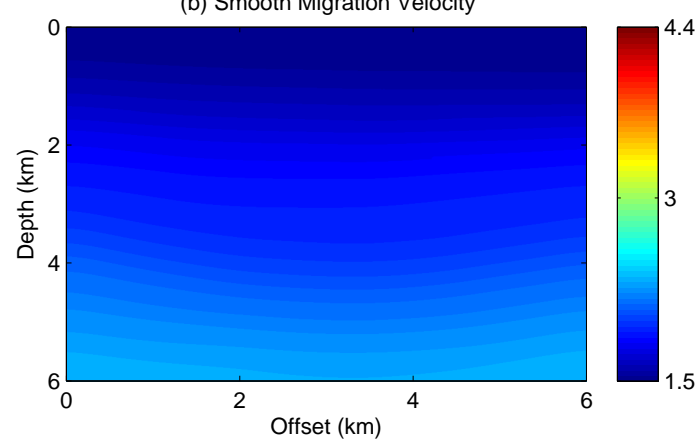

Figure 3: (a) A velocity model with a salt body on the left side; (b) The smooth migration velocity model without the salt body.

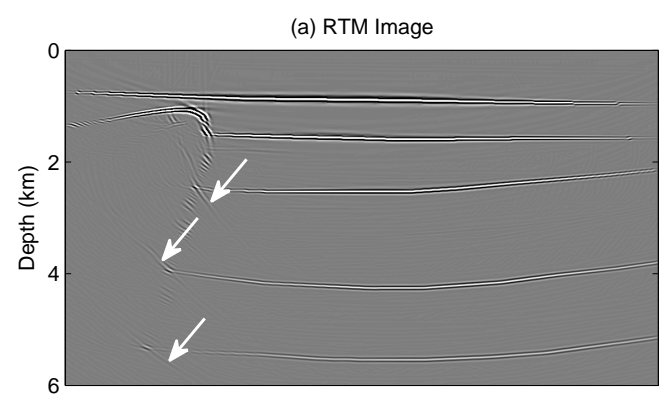

(b) RTM of Prism Waves

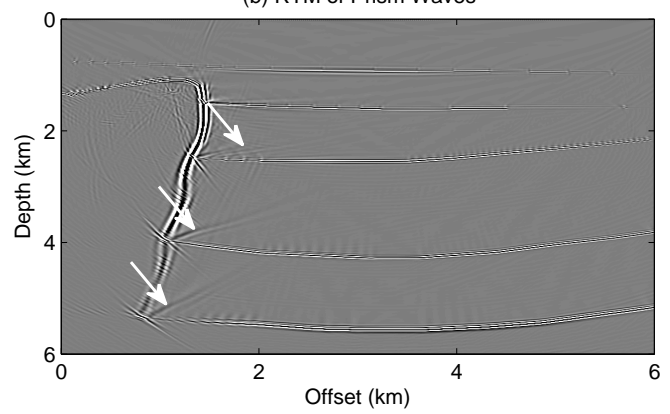

Figure 4: (a) The RTM image obtained with the smooth migration velocity model. Along the salt boundary, only a few diffractors are visible; (b) The RTM image of the prism waves with the same velocity model. The irregular salt boundary is well imaged. White arrows point to artifacts.

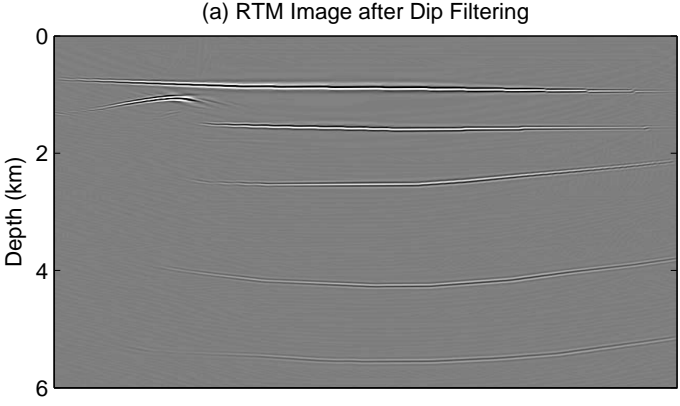

(b) RTM of Prism Waves

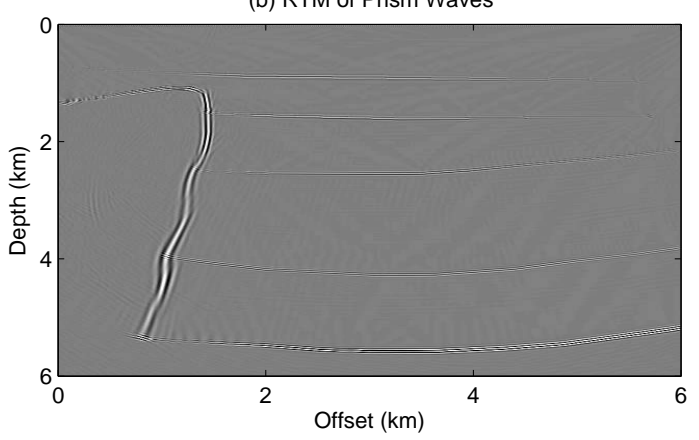

Figure 5: (a) The RTM image obtained with the smooth migration velocity model after dip filtering to keep subhorizontal reflectors only; (b) The RTM image of the prism waves.

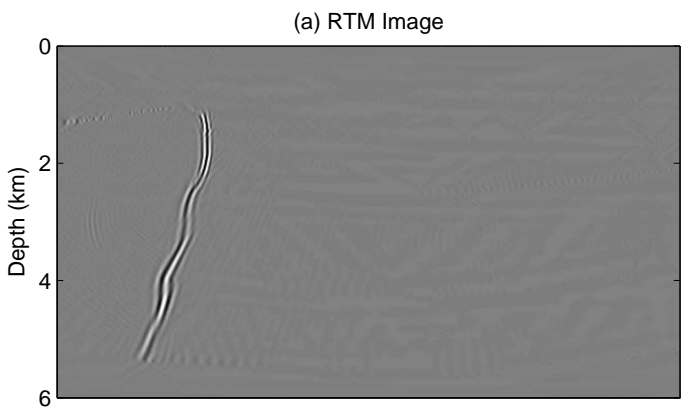

(b) Final Image

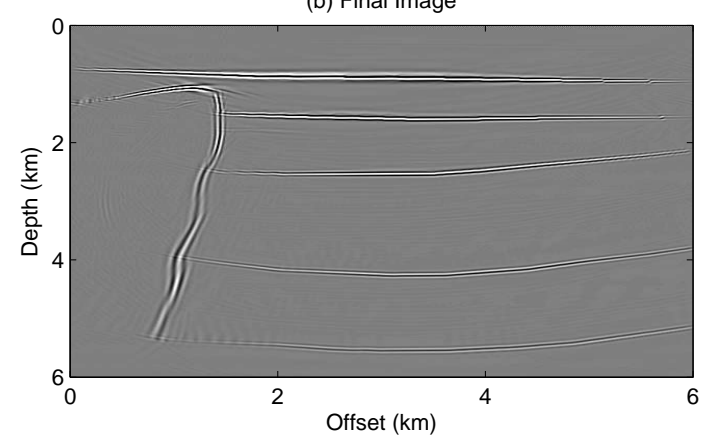

Figure 6: (a) The RTM image of the prism waves after dip filtering for subvertical reflectors only; (b) The sum of two partial images: one from conventional RTM and one from migration of the prism waves. 
http://dx.doi.org/10.1190/segam2013-0414.1

\section{EDITED REFERENCES}

Note: This reference list is a copy-edited version of the reference list submitted by the author. Reference lists for the 2013 SEG Technical Program Expanded Abstracts have been copy edited so that references provided with the online metadata for each paper will achieve a high degree of linking to cited sources that appear on the Web.

\section{REFERENCES}

Baysal, E., D. D. Kosloff, and J. W. C. Sherwood, 1983, Reverse time migration: Geophysics, 48, 1514 1524, http://dx.doi.org/10.1190/1.1441434.

Beylkin, G., 1985, Imaging of discontinuities in the inverse scattering problem by inversion of a causal generalized Radon transform: Journal of Mathematical Physics, 26, no. 1, 99-108, http://dx.doi.org/10.1063/1.526755.

Hale, D., N. R. Hill, and J. Stefani, 1992, Imaging salt with turning seismic waves: Geophysics, 57, 1453-1462, http://dx.doi.org/10.1190/1.1443213.

Jones, I. F., M. C. Goodwin, I. D. Berranger, H. Zhou, and P. A. Farmer, 2007, Application of anisotropic 3D reverse time migration to complex North Sea imaging: 77th Annual International Meeting, SEG, Expanded Abstracts, 2140-2144.

Liu, F., G. Zhang, S. A. Morton, and J. P. Leveille, 2011, An effective imaging condition for reverse-time migration using wavefield decomposition: Geophysics, 76, no. 1, S29-S39, http://dx.doi.org/10.1190/1.3533914.

Luo, Y., and G. T. Schuster, 1991, Wave-equation traveltime inversion: Geophysics, 56, 645-653, http://dx.doi.org/10.1190/1.1443081.

McMechan, G. A., 1983, Migration by extrapolation of time-dependent boundary values: Geophysical Prospecting, 31, 413-420, http://dx.doi.org/10.1111/j.1365-2478.1983.tb01060.x.

Ratcliff, D. W., S. H. Gray, and N. D. Whitmore, Jr., 1991, Seismic imaging of salt structures in the Gulf of Mexico: 61st Annual International Meeting, SEG, Expanded Abstracts, 1164-1165.

Ratcliff, D. W., S. H. Gray, and N. D. Whitmore, Jr., 1992, Seismic imaging of salt structures in the Gulf of Mexico: The Leading Edge, 11, 15-31, http://dx.doi.org/10.1190/1.1436876.

Stolt, R. H., and A. K. Benson, 1986, Seismic migration: Theory and practice: Geophysical Press.

Whitmore, N. D., 1983, Iterative depth migration by backward time propagation: 53rd Annual International Meeting, SEG, Expanded Abstracts, 382-385, http://dx.doi.org/10.1190/1.1893867. 